\title{
ZETA FUNCTIONS OF WEIGHTED GRAPHS AND COVERING GRAPHS
}

\author{
MATTHEW D. HORTON, H. M. STARK, AND AUDREY A. TERRAS
}

\begin{abstract}
We find a condition for weights on the edges of a graph which insures that the Ihara zeta function has a 3-term determinant formula. Then we investigate the locations of poles of abelian graph coverings and compare the results with random covers. We discover that the zeta function of the random cover satisfies an approximate Riemann hypothesis while that of the abelian cover does not.
\end{abstract}

\section{INTRODUCTION}

Acknowledgements: ${ }^{12}$.

The idea of weights associated to an edge of a graph is a natural one in applications such as

- electrical networks: resistance, capacitance in a wire corresponding to an edge;

- random walks: probability to move along a given edge;

- quantum graphs: each edge is an interval with a given length and a Schrödinger equation (see [5]).

We considered the Ihara zeta function $\zeta(u, X, v)$ of a (finite) weighted graph $X$ with weight $v$ in our paper [8]. In Section 2 of the present paper, the first object is to see whether there is a condition for edge weights of a graph which will allow us to prove a weighted analog of the Ihara three term determinant formula for the zeta function. We will find such a condition. We require $v\left(e^{-1}\right)=2-v(e)$, where $e^{-1}$ denotes the edge in the opposite direction from directed edge $e$. This condition allows us to prove the weighted analog of the Ihara determinant formula. See Theorem 1 . The theorem involves an adjacency matrix that is a function of the complex variable $u$. One might ask whether graphs arising in nature satisfy the condition $v\left(e^{-1}\right)=2-v(e)$, however.

At the end of Section 2, we will find an analog of the prime number theorem for graphs with positive integer edge weights satisfying the perhaps more natural condition that $v\left(e^{-1}\right)=v(e)$.

In Section 3, we consider abelian unweighted graph coverings. An abelian unweighted graph covering $Y$ over $X$ means that the covering is normal with abelian Galois group. For an $d$-sheeted covering to be normal, there must be $d$ covering isomorphisms (forming the Galois group) preserving the covering projection from $Y$ down to $X$. Section 3 begins with the observation that, for abelian graph coverings, the situation is similar to the case of weighted graphs satisfying the condition $v\left(e^{-1}\right)=2-v(e)$. In the two cases, one has the same sort of formula for the reciprocal of zeta as $\operatorname{det}(I-W)$, where $W$ is given either by Definition 6 or Definition 13. Moreover, the proof of the Ihara formula for the Artin-Ihara L-function corresponding to a character of an abelian Galois group is essentially the same as the proof of Theorem 1. There is some similarity in adjacency matrices. Compare Definition 7 with that in Theorem 4.

The main part of Section 3 gives the results of some of our experiments on the locations of poles of zeta functions of large abelian graph coverings. The question is: Do the pole locations for these abelian covers look anything like the pole locations for random graph coverings? For the $\mathbb{Z}_{m} \times \mathbb{Z}_{n}$ coverings of $X=2$ loops with an extra vertex on 1 loop which we consider, the graph theory analog of the Riemann hypothesis, formulated in [8] and Part III of [14], is visibly false (see Figure 7). However, for the example of a random cover of the same base graph considered here, the Riemann hypothesis appears to be approximately true (see Figure 9). The Riemann hypothesis for the Ihara zeta function $\zeta(u, X)$ of a graph $X$ says that if $R$ is the closest pole to 0 (necessarily positive) of $\zeta(u, X)$, then $\zeta(u, X)$ is pole-free when $R<|u|<\sqrt{R}$.

The reciprocal of the zeta function of an unweighted graph is $\operatorname{det}\left(I-u W_{0}\right)$, where $W_{0}$ is from Definition 4 . Thus our experiments outlined above are related to experiments on the spectrum of $W_{0}$ for large covers of a fixed base graph $X$ which have been performed by Angel, Hoory and Friedman [1] who call $W_{0}$ the "non-backtracking adjacency matrix". Moreover Angel, Hoory and Friedman have obtained a way of computing the spectrum of the analog of the $W_{0}$-edge matrix for the universal cover $\widetilde{X}$ of the base graph $X$. They conjecture that the new (i.e., not arising from the base graph) spectrum of the $W_{0}$-edge matrix for a random $d$-cover should approach this region as $d \rightarrow \infty$. This is related to Alon conjecture's for regular

Date: Sept. 19, 2007.

2000 Mathematics Subject Classification. 11M99, 05C25, 05C50.

Key words and phrases. Ihara zeta function, weighted graph, Artin L-function for abelian graph covering.

${ }^{1}$ The travel of A. Terras was partially supported by NSF grant DMS 0648786

${ }^{2}$ H. M. Stark and A. Terras would like to acknowledge the hospitality and support of the Newton Institute while writing this paper. 
graphs. See Friedman [4]. They note that $1 / \sqrt{R}$ is the spectral radius of the $W_{0}$ operator for $\tilde{X}$. Thus an approximate version of the Riemann hypothesis stated above is a consequence of the conjecture of Angel, Hoory, and Friedman for irregular covering graphs of a fixed base graph.

One can also ask whether the spectrum of the $W_{0}$-edge matrix has anything to do with the Girko circle law (see Bai [2]). Consider a set of random $n \times n$ real matrices with entries independent and with the standard normal distribution. Then, for $\lambda$ in the set of spectra of the matrices, as $n \rightarrow \infty$, the Girko circle law says that the quantity $\frac{\lambda}{\sqrt{n}}$ becomes uniformly distributed on the unit disk $\subset \mathbb{C}$. Our experiments, some of which appear in section 3 as figures 7 and 9 , do give rise to regions that appear to be 2-dimensional, but these regions are definitely not the entire unit disk. Of course, we are looking at the poles of $\zeta(u, Y)$ when $Y$ is a large cover of a small fixed base graph $X$. One wonders what would happen if $X$ itself were a large random graph. Also one wonders what happens for directed graphs or for zeta functions allowing backtracking. Note also that Figure 2 in [8] shows the poles of the Ihara zeta function for a random graph (as produced by Mathematica) and the poles are clustered near the Riemann hypothesis circle.

One can then ask whether there are Galois covers of a fixed base graph that mimic the behavior of random covers. One would not expect them to be abelian or even solvable covers of a fixed base graph if one expects things to be similar to the case of regular graphs. Here we refer to the construction of Ramanujan graphs by Margulies and independently by Lubotzky, Phillips and Sarnak [11]. The group involved in this latter paper, was the special linear group of $2 \times 2$ matrices with determinant 1 over a finite field. One might then ask whether such "random" covers exhibit any properties of expanders. Finally one would want to study the connections with quantum chaos. Is there some analog of the work on spacings of zeros of zetas for irregular graphs? See Terras [16] for some background on arithmetical quantum chaos.

The weighted zeta functions that we consider should be compared with those investigated by Smilansky in [12]. These zeta functions come from a similar $W$-matrix but this time allowing backtracking.

We wish to thank Joel Friedman, Shlomo Hoory, Uzy Smilansky, and Brian Winn for interesting discussions while we were writing this paper.

\section{Weighted GRAPHS AND THEIR ZETA FunCTIONS}

Let $X$ be a graph. We make our usual assumptions (finite, connected, undirected, not necessarily regular or simple). Suppose $X$ has $|V|$ vertices and $|E|$ undirected edges. Orient the edges arbitrarily and obtain $2|E|$ oriented edges labeled

$$
e_{1}, e_{2}, \cdots, e_{|E|}, e_{|E|+1}=e_{1}^{-1}, \ldots, e_{2|E|}=e_{|E|}^{-1} .
$$

Assume in this section that our graph $X$ comes with a given weight or length function $v(e)$ attached to directed edges $e$, or equivalently to pairs of vertices. Write $(X, v)$ to denote the weighted graph. Viewing the function $v$ as a length, it is natural to use it to define the length of a path or walk in $X$. A path $C$ in $X$ is a word in the directed edges: $C=a_{1} \cdots a_{s}$, where $a_{j}$ is a directed edge of $X$. We will write $e \in C$ if $e=a_{j}$ for some $j$.

Definition 1. The length of a path $C$ in $X$ is $v(C)=\sum_{e \in C} v(e)$.

It might be natural for some applications to replace the sum in the preceding definition by a product. Although the product variant fits easily into our multivariable edge matrix theory, we will not consider this possibility here.

The Ihara zeta function for a graph $X$ has been considered by many authors. In particular, see our paper [8] for a similar point of view. Other references are Bass [3], Hashimoto [6], Kotani and Sunada [9], Stark and Terras [14], and [17].

"Primes" $[P]$ in $X$ are equivalence classes of closed backtrackless tailless primitive paths $P$. Let a path $C$ have the form $C=a_{1} a_{2} \cdots a_{s}$, where $a_{j}$ is an oriented edge of $X$. The path $C$ is backtrackless if $a_{i+1} \neq a_{i}^{-1}$, for all $i$. Tailless means that $a_{s} \neq a_{1}^{-1}$. The equivalence class $[C]$ is the set

$$
[C]=\left\{a_{1} a_{2} \cdots a_{s}, a_{2} a_{3} \cdots a_{s} a_{1}, \ldots, a_{s} a_{1} \cdots a_{s-1}\right\} .
$$

$[P]$ is primitive means $P \neq D^{t}$, for any integer $t \geq 2$ and path $D$ in $X$. In short, a prime is an analog of a primitive closed geodesic in a Riemannian manifold. Length is minimized.

Here $r_{X}$ will denote the rank of the fundamental group of $X$. We have $r_{X}-1=|E|-|V|$. Then $r_{X}$ is the number of edges deleted from $X$ to form a spanning tree.

Our Usual Hypotheses.

We assume from now on that the graph $X$ is finite connected, with rank $r_{X}>1$, and that $X$ has no degree 1 vertices. If $(X, v)$ is a weighted graph, the Ihara zeta function of a weighted graph is defined as in [8]. 


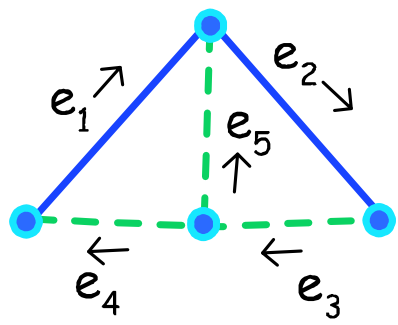

FIgURE 1. The edges of $K_{4}^{\prime}$ are labeled. Inverse edges are $e_{i+5}=e_{i}^{-1}$.

Definition 2. The Ihara zeta function of a weighted graph $(X, v)$ is defined, assuming all weights $v(e)$ are positive, for $|u|$ small and $u \notin(-\infty, 0)$, to be

$$
\zeta(u, X, v)=\prod_{[P]}\left(1-u^{v(P)}\right)^{-1} .
$$

Here the product is over all primes $[P]$ of $X$.

Let $0<\varepsilon \leq v(e)$, for all edges $e$. Then one can see that the infinite product for the weighted Ihara zeta function converges when $|u| \leq(2|E|)^{-1 / \varepsilon}$. If we do not assume that all weights are positive, there is a problem proving convergence of the infinite product.

Clearly when $v=1$, meaning the function such that $v(e)=1$ for all edges $e$ in $X$, we have $\zeta(u, X, 1)=\zeta(u, X)$, the original Ihara zeta function. If the weights are all positive integers and $v(e)=v\left(e^{-1}\right)$, we can realize the weighted zeta of $(X, v)$ as an original Ihara zeta of an unweighted inflated graph $X_{v}$. To create $X_{v}$, we just add $v(e)-1$ extra vertices on edge $e$ of $X$ and then view the new graph as unweighted. From the point of view of adjacency matrices, one might be tempted instead to add $v(e)-1$ edges between the starting and ending vertices of $e$. That would disastrously change the zeta function, however.

Example 1. Forget our assumption that the rank $r_{X}$ is greater than 1 and let $X=K_{3}$, a 3-cycle. Suppose that the edges are directed counterclockwise around the cycle. We write the weights associated to the various edges as the letters of the alphabet in order. It follows that

In this case there are only 2 primes.

$$
\zeta(u, X, v)=\left(1-u^{a+b+c}\right)\left(1-u^{d+e+f}\right)
$$

Example 2. Let $X=K_{4}^{\prime}$, the graph obtained by deleting an edge from $K_{4}$, with the edges directed as in Figure 1 . We write the weights associated to the various edges as the letters of the alphabet in order.

Using formula (2.8) below and Scientific Workplace, one sees that for weights $v=(a, b, c, d, e, f, g, h, i, j)$, we have $\zeta\left(u, K_{4}^{\prime}, v\right)^{-1}=$

$$
\operatorname{det}\left(\begin{array}{cccccccccc}
-1 & u^{a} & 0 & 0 & 0 & 0 & 0 & 0 & 0 & u^{a} \\
0 & -1 & u^{b} & 0 & 0 & 0 & 0 & 0 & 0 & 0 \\
0 & 0 & -1 & u^{c} & u^{c} & 0 & 0 & 0 & 0 & 0 \\
u^{d} & 0 & 0 & -1 & 0 & 0 & 0 & 0 & 0 & 0 \\
0 & u^{e} & 0 & 0 & -1 & u^{e} & 0 & 0 & 0 & 0 \\
0 & 0 & 0 & 0 & 0 & -1 & 0 & 0 & u^{f} & 0 \\
0 & 0 & 0 & 0 & 0 & u^{g} & -1 & 0 & 0 & u^{g} \\
0 & 0 & 0 & 0 & 0 & 0 & u^{h} & -1 & 0 & 0 \\
0 & 0 & 0 & 0 & u^{i} & 0 & 0 & u^{i} & -1 & 0 \\
0 & 0 & 0 & u^{j} & 0 & 0 & 0 & u^{j} & 0 & -1
\end{array}\right) .
$$

Next consider what happens if the weights satisfy the condition that leads to an Ihara formula for zeta: $v_{1}\left(e_{j}^{-1}\right)=2-v_{1}\left(e_{j}\right)$, for all edges $e_{j}$. Then $\zeta\left(u, K_{4}^{\prime}, v_{1}\right)^{-1}=$ 


$$
=\frac{1}{u^{e} u^{a} u^{b} u^{c} u^{d}}\left(\begin{array}{c}
u^{10} u^{e}-u^{8} u^{e}-u^{6} u^{a} u^{d}+u^{10} u^{a} u^{d}-u^{4} u^{2 e} u^{b} u^{c}+u^{8} u^{2 e} u^{b} u^{c} \\
-u^{2} u^{2 a} u^{2 d} u^{b} u^{c}+u^{6} u^{2 a} u^{2 d} u^{b} u^{c}+u^{e} u^{a} u^{b} u^{c} u^{d}-u^{2 e} u^{2 b} u^{2 c} u^{a} u^{d} \\
-u^{2 a} u^{2 b} u^{2 c} u^{2 d} u^{e}+u^{4} u^{2 e} u^{2 b} u^{2 c} u^{a} u^{d}+u^{2} u^{2 a} u^{2 b} u^{2 c} u^{2 d} u^{e} \\
+2 u^{6} u^{e} u^{a} u^{b} u^{c} u^{d}+u^{8} u^{e} u^{a} u^{b} u^{c} u^{d}-4 u^{10} u^{e} u^{a} u^{b} u^{c} u^{d}
\end{array}\right) .
$$

If instead the weights satisfy the more natural condition of the prime number theorem when the weights are positive integers: $v_{2}\left(e_{j}^{-1}\right)=v_{2}\left(e_{j}\right)$, for all edges $e_{j}$, then $\zeta\left(u, K_{4}^{\prime}, v_{2}\right)^{-1}=$

$$
\begin{aligned}
= & u^{2 e} u^{2 a} u^{2 d}-2 u^{e} u^{b} u^{c}-2 u^{e} u^{a} u^{d}+u^{2 e} u^{2 b} u^{2 c}+u^{2 a} u^{2 b} u^{2 c} u^{2 d} \\
& -2 u^{a} u^{b} u^{c} u^{d}+2 u^{2 a} u^{2 d} u^{e} u^{b} u^{c} \\
& +2 u^{2 b} u^{2 c} u^{e} u^{a} u^{d}-4 u^{2 e} u^{2 a} u^{2 b} u^{2 c} u^{2 d}+2 u^{2 e} u^{a} u^{b} u^{c} u^{d}+1 .
\end{aligned}
$$

Now recall another determinant formula for the unweighted zeta. First we make the definition of the adjacency matrix $A_{X}$ and the $Q_{X}$ matrix for this case.

Definition 3. For an unweighted graph $X$, the adjacency matrix $A_{X}$ is an $n \times n$ matrix with $i, j$ entry equal to the number of edges from vertex $i$ to vertex $j$ if $i \neq j$ and twice the number of loops at vertex $i$ if $i=j$. The degree -1 matrix $Q_{X}$ is the diagonal matrix whose $j$ th diagonal entry is $(-1+$ degree of $j$ th vertex $)$.

The generalization of Ihara's formula (proved for irregular graphs by Hashimoto and Bass) says

$$
\zeta(u, X, 1)^{-1}=\left(1-u^{2}\right)^{r_{X}-1} \operatorname{det}\left(I-A_{X} u+Q_{X} u^{2}\right),
$$

where $r_{X}=|E|-|V|+1=m-n+1$ is the rank of the fundamental group of $X$. We gave a version of Bass's proof of Ihara's formula in [8].

Question: Can we generalize formula (2.3) to weighted graphs?

To do this, we need to use the basic facts about the edge zeta function from [8].

Definition 4. The edge matrix $W$ for graph $X$ is a $2|E| \times 2|E|$ matrix of complex numbers, with $a, b$ entry corresponding to the oriented edges $a$ and $b$. This $a, b$ entry is the complex variable $w_{a b}$ if edge $a$ feeds into edge $b$ and $b \neq a^{-1}$ and the $a, b$ entry is 0 otherwise. Define the 0,1 edge matrix $W_{0}$ by setting all non-0 entries of $W$ equal to 1.

Angel, Friedman and Hoory [1] call $W_{0}$ the "non-backtracking adjacency matrix." Given a path $C$ in $X$, which is written as a product of oriented edges $C=a_{1} a_{2} \cdots a_{S}$, the edge norm of $C$ is

$$
N_{E}(C)=w_{a_{1} a_{2}} w_{a_{2} a_{3}} \cdots w_{a_{s-1} a_{s}} w_{a_{s} a_{1}} .
$$

Definition 5. The edge Ihara zeta function is defined for sufficiently small $\left|w_{e f}\right|$ by

$$
\zeta_{E}(W, X)=\prod_{[P]}\left(1-N_{E}(P)\right)^{-1},
$$

where the product is over primes in $X$.

Definition 6. If $(X, v)$ is a weighted graph with weight function $v$, define the $2|E| \times 2|E|$ diagonal matrix $R_{v}$ to have diagonal entry corresponding to oriented edge e given by $u^{\nu(e)-1}$. If $W_{0}$ is the 0,1 edge matrix from Definition 4 , the weighted graph $W-$ matrix $W_{v}$ is defined to be

$$
W_{v}=u R_{v} W_{0}
$$

Here we assume that $u$ is a complex variable with $u \notin(-\infty, 0]$.

Note that $W_{1}=u W_{0}$. In [8], we wrote $W_{1}$ for what we now call $W_{0}$. This change of notation seems necessary, if we are to write $W_{v}$ as in formula (2.5). Now plug the $W_{v}$ formula (2.5) into the edge zeta and you get the weighted Ihara zeta function ; i.e.,

$$
\zeta_{E}\left(W_{\nu}, X\right)=\zeta(u, X, v)
$$

Equivalently, you could specialize $w_{a b}=u^{(v(a)+v(b)) / 2}$.

From [8], we have the simplest determinant formula for the edge zeta function

$$
\zeta_{E}(W, X)^{-1}=\operatorname{det}(I-W) \text {. }
$$

From formulas (2.5), (2.6), and (2.7), we immediately obtain the simplest determinant formula for the weighted Ihara zeta function with $W_{v}$ as in Definition 6:

$$
\zeta(u, X, v)^{-1}=\operatorname{det}\left(I-W_{v}\right)
$$


We used formula (2.8) to compute the weighted zeta in Example 2 above.

Now the question is: how can we generalize the Ihara type formula (2.3) to weighted graphs? For this we need a restriction on the weights. There are $\mathbf{2}$ conditions on the weights that we will assume in order to obtain an Ihara formula.

Condition 1. The weight satisfies $v\left(e^{-1}\right)=2-v(e)$.

This condition may not appear to be so natural, but we need it to obtain a 3-term Ihara determinant formula for the zeta function. If in addition, we want to show that the infinite product defining zeta converges, we must avoid having negative weights and thus must assume another condition.

Condition 2. The weight satisfies $0<v(e)<2$.

Note that if we want $v\left(e^{-1}\right)=2-v(e)=v(e)$, then we need $v(e)=1$, for all edges, the case of the original Ihara zeta function.

To give an Ihara 3-term determinant formula for our weighted graph, we need to define a version of the adjacency matrix which depends on the variable $u$.

Definition 7. The adjacency matrix $A_{\nu}$ for the weighted graph $(X, v)$ is defined as the $|V| \times|V|$ matrix with a, $b$ entry

$$
\left(A_{v}\right)_{a, b}=\sum_{\substack{e \\ a \rightarrow b}} u^{\nu(e)-1}
$$

where the sum is over edges e starting at vertex $a$ and ending at vertex $b$.

Assuming $v\left(e^{-1}\right)=2-v(e)$, this adjacency matrix is only symmetric if $v(e)=1$, for all edges $e$. In this case, it is the usual adjacency matrix. The adjacency matrix is Hermitian if $|u|=1$, again assuming $v\left(e^{-1}\right)=2-v(e)$.

Example 3. $K_{4}^{\prime}=K_{4}$ minus an edge, with weights $v\left(e_{j}\right)=\frac{j}{5}=2-v\left(e_{j}^{-1}\right), j=1,2,3,4,5$.

Number the vertices starting at the leftmost and proceeding clockwise in Figure 1. Then the adjacency matrix is

$$
A_{v}=\frac{1}{u}\left(\begin{array}{cccc}
0 & u^{\frac{1}{5}} & 0 & u^{\frac{6}{5}} \\
u^{\frac{9}{5}} & 0 & u^{\frac{2}{5}} & u \\
0 & u^{\frac{8}{5}} & 0 & u^{\frac{3}{5}} \\
u^{\frac{4}{5}} & u & u^{\frac{7}{5}} & 0
\end{array}\right)
$$

It follows that

$$
\zeta\left(u, K_{4}^{\prime}, v\right)^{-1}=\left(1-u^{2}\right)(u-1)(u+1)\left(2 u^{3}+u+1\right)\left(2 u^{3}+u-1\right) .
$$

The shock is that, despite the fractional weights, we once more have the reciprocal of a polynomial. The reader should then attempt to check that the paths in the graph have integer lengths. One wonders what the factorization means in the context of Part II of [14].

Next we change the weight on the first edge.

Example 4. $K_{4}^{\prime}=K_{4}$ minus an edge, with the weights of the previous example except that $v\left(e_{1}\right)=\frac{2}{5}=2-v\left(e_{1}^{-1}\right)$.

Now

$$
\zeta\left(u, K_{4}^{\prime}, v\right)^{-1}=\left(1-u^{2}\right)\left(2 u^{6}-u^{4}+4 u^{8}-2 u^{\frac{11}{5}}-u^{\frac{19}{5}}-u^{\frac{21}{5}}-2 u^{\frac{29}{5}}+1\right) .
$$

The inverse zeta is no longer a polynomial. It has a positive real root at approximately $u=0.60407$.

Example 5. $K_{4}^{\prime}=K_{4}$ minus an edge, with the weights $v\left(e_{1}\right)=\frac{1}{2}, v\left(e_{2}\right)=1, v\left(e_{3}\right)=\frac{5}{4}, v\left(e_{4}\right)=\frac{5}{4}, v\left(e_{5}\right)=\frac{3}{4}$, and $v\left(e_{i}^{-1}\right)=2-v\left(e_{i}\right), i=1,2, \ldots, 5$.

Then

$$
\zeta\left(u, K_{4}^{\prime}, v\right)=\zeta\left(u, K_{4}^{\prime}, 1\right)=\left(1-u^{2}\right)(u-1)\left(2 u^{2}+u+1\right)\left(u^{2}+1\right)\left(2 u^{3}+u^{2}-1\right) .
$$

This is also a surprise at first. The weighted zeta equals the unweighted zeta. Since $v(e)+v\left(e^{-1}\right)=2$, it suffices to check that $v(P)=$ the number of edges in $P$, for closed paths $P$ generating the fundamental group of $K_{4}^{\prime}$. Thus it suffices to check this for $P=e_{1} e_{5}^{-1} e_{4}$ and for $P=e_{2} e_{3} e_{5}$, using the labeling in Figure 1. Alternatively, we can use the (multi-) path zeta function of [14] and [8]. It requires specializing the $z$-variables as in formula (18) on page 181 of Part II of [14] or formula (4.1) on page 187 of [8]. Then one specializes the $w$-variables as in Definition 6 . The resulting $4 \times 4$ matrix for our example is not the same as the matrix for the unweighted graph, but it is similar to that matrix. 
Example 6. One can similarly consider the weighted graph consisting of 2 loops on a single vertex with weights a on loop 1, $b$ on loop 2 and $2-a, 2-b$ on the inverse edges, respectively. Then it is easily seen that

$$
\zeta\left(u, K_{4}^{\prime}, v\right)^{-1}=\left(1-u^{2}\right)\left(3 u^{2}+1-\left(u^{a}+u^{2-a}+u^{b}+u^{2-b}\right)\right) .
$$

If we set $a=x, b=1$, for $0<x<2$, we find that the smallest positive root of the inverse zeta approaches 0 as $x \rightarrow 0$.

Note that

$$
\left(\left[\frac{d\left(u A_{v}\right)}{d u}\right]_{u=1}\right)_{v, w}=\sum_{\substack{e \\ v \rightarrow w}} v(e) .
$$

Here the sum is over the directed edges $e$ with initial vertex $v$ and terminal vertex $w$. This is the usual adjacency matrix of a weighted graph.

Next we imitate the Bass proof of the Ihara 3 term determinant formula given in [8]. As in that paper, set $J=\left(\begin{array}{c}0 \\ I_{|E|} \\ 0\end{array}\right)$. Then define the $|V| \times 2|E|$ start matrix $S$ and the $|V| \times 2|E|$ terminal matrix $T$ by setting

$$
s_{v e}=\left\{\begin{array}{cc}
1, & \text { if } v \text { is starting vertex of edge } e, \\
0, & \text { otherwise, }
\end{array}\right.
$$

and

$$
t_{v e}=\left\{\begin{array}{cc}
1, & \text { if } v \text { is terminal vertex of edge } e, \\
0, & \text { otherwise. }
\end{array}\right.
$$

The matrix $Q$ is as before in Definition 3. The matrices $R_{v}$ and $W_{v}$ are from Definition 6 . The weighted adjacency matrix $A_{v}$ is from Definition 7.

Proposition 1. (Some Matrix Identities) Using the preceding definitions, the following formulas hold. We write ${ }^{t} M$ for the transpose of the matrix $M$. We always assume the weight $v$ satisfies Conditions 1 and 2 . This means $v\left(e^{-1}\right)=2-v(e)$ and all weights are positive.

1) $W_{v}=u R_{v} W_{0}$.

2) $A_{v}=S R_{v}^{t} T$.

3) $S J=T, \quad T J=S, Q+I_{|V|}=S^{t} S=T^{t} T$.

4) $W_{v}+u R_{v} J=u R_{v}{ }^{t} T S$.

5) $\left(R_{v} J\right)^{2}=I$.

Proof. 1) One sees easily from the definitions that

$$
\left(W_{\nu}\right)_{e, f}=u^{\nu(e)}\left(W_{0}\right)_{e, f}=\left(u R_{v} W_{0}\right)_{e, f} .
$$

2) Consider

$$
\left(S R_{v}{ }^{t} T\right)_{a, b}=\sum_{e} s_{a, e} u^{\nu(e)-1} t_{b, e} .
$$

The right hand side is a sum over edges $e$ such that $a$ is the initial vertex and $b$ is the terminal vertex of $e$, which is the $a, b$ entry of $A_{v}$ in Definition 7.

3) See [8] or part II of [14]. Note that we are using the labeling in formula (2.1).

4) Multiply the analogous formula for unweighted graphs on p. 186 of [8] by $u R_{v}$

5) Since we have assumed $v\left(e^{-1}\right)=2-v(e), R_{v}$ has the form

$$
\left(\begin{array}{cc}
U & 0 \\
0 & U^{-1}
\end{array}\right) \text {. }
$$

It is then easy to check the formula.

Now we can prove the Ihara determinant formula for weighted graphs.

Theorem 1. A Weighted Graph Version of Ihara's Theorem. With Definition 7 of the adjacency matrix $A_{v}$ of a weighted graph and assuming that the weight $v$ satisfies conditions 1 and 2 (i.e., $v\left(e^{-1}\right)=2-v(e)$ and all weights are positive), we have

$$
\zeta(u, X, v)^{-1}=\left(1-u^{2}\right)^{r-1} \operatorname{det}\left(I-A_{v} u+Q u^{2}\right)
$$


Proof. We follow the method of Bass as developed in Part II of [14], proceeding in a similar way to the case of abelian groups when all irreducible group representations are one-dimensional. In the following identity all block matrices are $(|V|+2|E|) \times$ $(|V|+2|E|)$, where the 1st block is $|V| \times|V|$. Use the preceding proposition to see that

$$
\begin{gathered}
\left(\begin{array}{cc}
I_{|V|} & 0 \\
R_{\nu}{ }^{t} T & I_{2|E|}
\end{array}\right)\left(\begin{array}{cc}
\left(1-u^{2}\right) I_{|V|} & u S \\
0 & I_{2|E|}-W_{v}
\end{array}\right) \\
=\left(\begin{array}{cc}
I_{|V|}-u A_{v}+u^{2} Q & u S \\
0 & I_{2|E|}+u R_{\nu} J
\end{array}\right)\left(\begin{array}{cc}
I_{|V|} & 0 \\
R_{v}{ }^{t} T-u^{t} S & I_{2|E|}
\end{array}\right) .
\end{gathered}
$$

Then we have an identity for $2 m \times 2 m$ matrices

$$
I_{2|E|}+u R_{\nu} J=\left(\begin{array}{cc}
I_{|E|} & u U \\
u U^{-1} & I_{|E|}
\end{array}\right)=\left(\begin{array}{cc}
I_{|E|} & 0 \\
u U^{-1} & I_{|E|}
\end{array}\right)\left(\begin{array}{cc}
I_{|E|} & u U \\
0 & \left(1-u^{2}\right) I_{|E|}
\end{array}\right) .
$$

Take the determinant of formula (2.9) to complete the proof using formula (2.10) and the fact that $|E|-|V|=r-1$.

Remark 1. Given a graph $X$ with positive integer weights $v$ satisfying $v(e)=v\left(e^{-1}\right)$, define $\pi_{v}(m)$ to be the number of primes $[P]$ in $X$ such that $v(P)=m$. Assume that the rank of the fundamental group $r$ is greater than 1 . Assume the weights are all positive integers such that $v(e)=v\left(e^{-1}\right)$. The number $R$ is the closest pole of $\zeta(u, X, v)$ to the origin. If $\Delta$ is the g.c.d. of the lengths of primes in $X$ and $\Delta$ divides $m$, the prime number theorem says

$$
\pi_{v}(m) \sim \frac{\Delta}{m} R^{-m}, \text { as } m \rightarrow \infty .
$$

To see why this is true, recall that the unweighted graph $X_{v}$, is obtained from $X$ by adding $v(e)-1$ extra vertices on each edge $e$ of $X$. There is a 1-1 correspondence between primes [P] in $X$ and primes $[\varphi(P)]$ in $X_{v}$. The length of the prime $\varphi(P)$ in the unweighted graph is the same as $v(P)$. The zeta function $\zeta\left(u, X_{v}, 1\right)$ is the same as $\zeta(u, X, v)$. Moreover the number $\pi_{\nu}(m)$ of primes $[P]$ in $X$ such that $\nu(P)=m$ is the same as the number $\pi_{1}(m)$ of primes $[\varphi(P)]$ in $X_{\nu}$ of ordinary length $m$. Note also that the greatest common divisor of lengths of primes in $X$ is the same as the g.c.d. of lengths of primes in $X_{\nu}$. And, finally, $R$, the closest pole of $\zeta(u, X, v)$ to the origin is the same as that for $\zeta\left(u, X_{v}, 1\right)$. The prime number theorem for the weighted graph $X$ follows from that for $X_{\nu}$ proved in [8], pages 181-182.

Note that the prime equivalence class $[P]$ from formula (2.2) in the weighted graph $X$ has fewer elements in general than the equivalence class $[\varphi(P)]$ in the unweighted graph $X_{\nu}$. Thus the generating function formula from $u \frac{d}{d u} \log \zeta(u, X, \nu)$ has a slight difference from the unweighted formula. See formula (2.13) in [8], page 182.

This leaves us with many questions. Is there a prime number theorem for graphs with arbitrary positive weights satisfying condition 1? We leave this question for a future work.

\section{Zeta Functions of Abelian Coverings of Unweighted Graphs}

Here we consider normal graph coverings with abelian Galois group. The general theory is worked out in Part II of [14], . See also [17].

From now on we consider only unweighted graphs $X$ and $Y$. However, we will find that many of the methods of the preceding section still apply and lead to analogs of Proposition 1 and Theorem 1.

Consider an unramified finite covering graph $Y$ of $X$. If our graphs had no multiple edges or loops, this would simply mean that there is a covering map $\pi: Y \longrightarrow X$ such that $\pi$ is an onto graph map and for each $x \in X$ and each $y \in \pi^{-1}(x)$, the set of points adjacent to $y$ in $Y$ is mapped by $\pi 1-1$, onto the set of points adjacent to $x$ in $X$. However, to make the fundamental theorems of Galois theory work in general, we need a more precise definition.

Each edge of $X$ is arbitrarily assigned a direction. A covering map $\pi$ not only takes vertices of $Y$ to vertices of $X$ but also takes edges of $Y$ to edges of $X$. Directions are then assigned to edges of $Y$ so that $\pi$ gives a direction preserving map of edges. Further it is required that $\pi$ gives an isomorphism between neighborhoods of $Y$ and neighborhoods of $X$. This is necessary to handle loops in $X$. For instance in Figure 2, "circular neighborhoods" of a selected vertex of $X$ and the three vertices of $Y$ projecting down to it are shown. Within the neighborhood of the vertex on $X$, a loop yields both an "outbound" edge and "inbound" edge in the neighborhood. Thus in each of the three neighborhoods of the lifted vertex in $Y$, there should be both outbound and inbound edges projecting to the loop.

If $Y$ has $d$ sheets, then there are at most $d$ distinct covering automorphisms.

Definition 8. A normal d-sheeted (unramified) graph covering $Y$ of $X$ is an d-sheeted graph covering with d covering automorphisms $\sigma: Y \longrightarrow Y$. The Galois group $G=\operatorname{Gal}(Y / X)$ is the set of all these $\sigma^{\prime}$ s. 


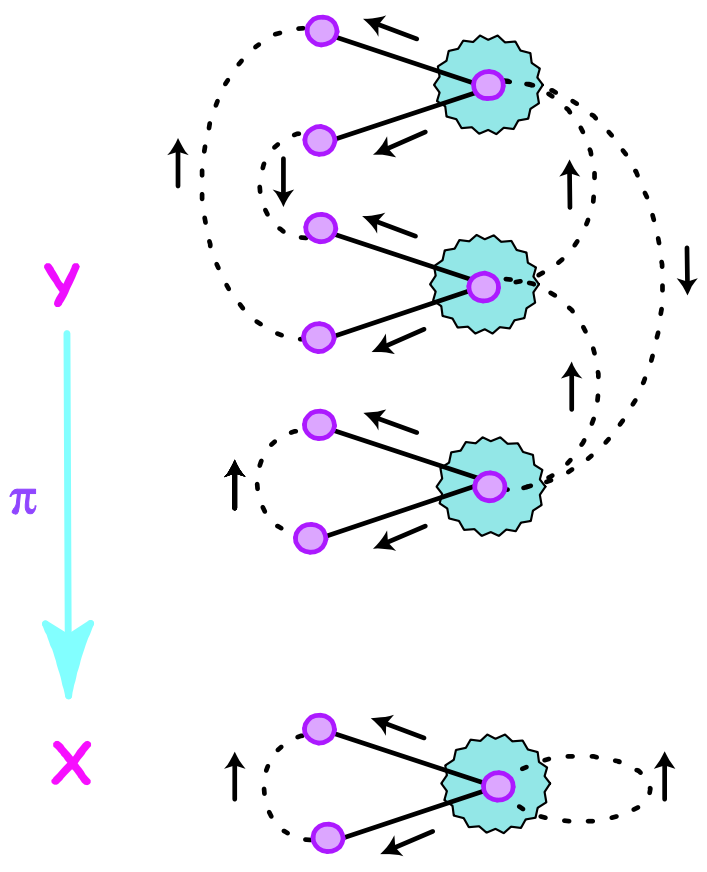

FIgURE 2. A 3-sheeted covering. The fuzzy area in $X$ is a neighborhood of a selected vertex. The 3 fuzzies in $Y$ are all of its inverse images under the projection $\pi$.

Example 7. The cube is a quadratic cover of the tetrahedron. See Figure 3. The dashed lines represent a spanning tree in the tetrahedron. The cube has 2 copies of this tree forming the 2 sheets of the covering. This is a Galois covering with Galois group of order 2.

In general one obtains $d$-sheeted unramified normal covers $Y$ of $X$ by taking $d$ copies of the spanning tree for $X$; the vertices of $Y$ are labeled $(x, \sigma), x \in X, \sigma \in \operatorname{Gal}(Y / X)$. An element $\tau \in \operatorname{Gal}(Y / X)$ acts on vertices of $Y$ via $\tau(x, \sigma)=(x, \tau \sigma)$. And

$$
\tau \text { (path from }(a, \lambda) \text { to }(b, \sigma))
$$

is the path from $(a, \tau \lambda)$ to $(b, \tau \sigma)$. A permutation representation of the Galois group $\operatorname{Gal}(Y / X)$ tells us how to lift edges of $X$ to $Y$. Of course we only need to think about the edges of $X$ left out of a spanning tree (i.e., the edges corresponding to generators of the fundamental group of $X$ ).

In Part II of [14] we show that there are analogs of all the basic theorems of Galois theory. In particular, there is a 1-1 correspondence between subgroups $H$ of $\operatorname{Gal}(Y / X)$ and intermediate covers $\widetilde{X}$ to $Y / X$. Of course, the concept of intermediate cover needs a careful definition. For example, given $H$ a subgroup of $\operatorname{Gal}(Y / X)$, the points of the top graph $Y$ have the form $(x, \sigma), x \in X, \sigma \in \operatorname{Gal}(Y / X)$. The points of the intermediate graph $\widetilde{X}$ have the form $(x, H \sigma), x \in X, H \sigma \in H \backslash G$. There is an edge between $(x, H \lambda)$ and $(u, H \sigma)$ in $\widetilde{X}$ iff there are $h, h^{\prime}$ in $H$ such that $(x, h \lambda)$ and $\left(u, h^{\prime} \sigma\right)$ have an edge between them in $Y$.

There are also analogs of the facts about the splitting of prime ideals in extensions of algebraic number fields.

Definition 9. Let $Y / X$ be a d-sheeted unramified finite normal cover. Suppose that the projection map is $\pi: Y \longrightarrow X$ and that $[D]$ is a prime in $Y$. Then for some $f \geq 1, \pi(D)=C^{f}$, where $[C]$ is a prime of $X$. We say $[D]$ is a prime of $Y$ over the prime $[C]$ of $X$. We call $f=f(Y / X, D)=$ the residual degree of $D$ with respect to $Y / X$. And we set $g=g(Y / X, D)=$ the number of primes $[D]$ in $Y$ over $[C]$ in $X$. In this paper, $e=e(Y / X, D)=$ ramification index $=1$.

As in number theory, if $Y / X$ is a normal covering, we have the formula

$$
\text { efg }=d \text {. }
$$

Example 8. Splitting of Primes in the Cube/K 4 . Look at Figures 4 and 5.

In Figure 4, there are two prime cycles $R^{\prime}$ and $R^{\prime \prime}$ in the cube over the prime cycle $R$ in the tetrahedron. In this case $f=1, g=2, e=1$.

In Figure 5, there is only one prime cycle $P^{\prime}$ in the cube over the prime cycle $P$ in the tetrahedron. For this case, $f=2$, since $P^{\prime}$ is the lift of $P^{2}$ and $g=1$. It is always true that the ramification index $e=1$. 

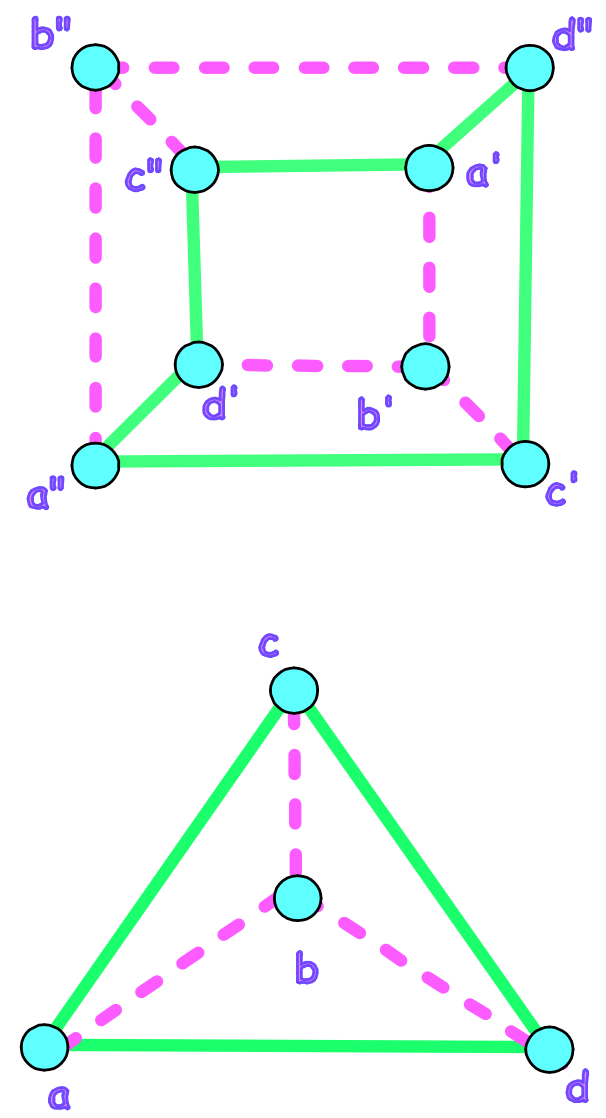

FIgURE 3. The cube is a quadratic covering of the tetrahedron. A spanning tree for the tetrahedron is indicated with dashed lines. Two copies of this tree are seen in the cube.
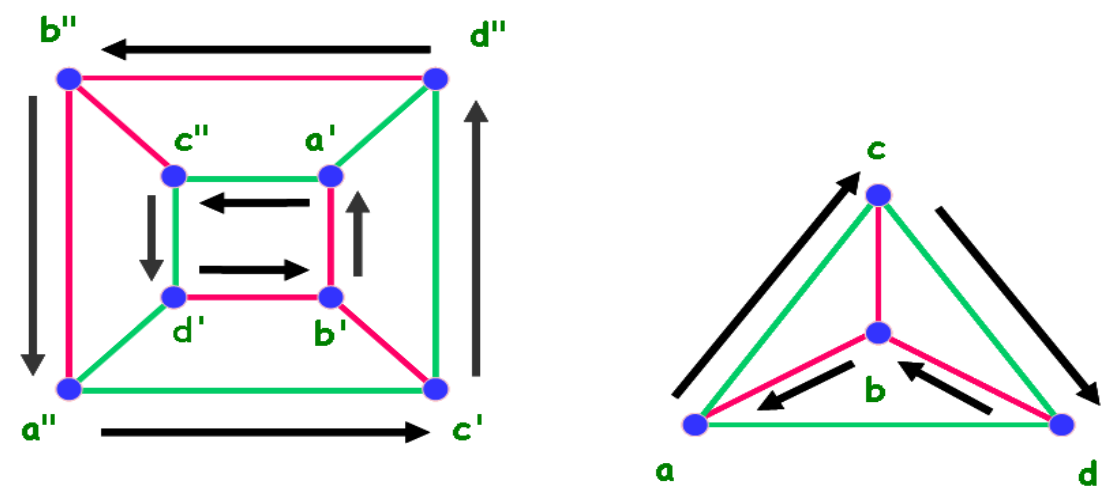

FIgURE 4. Picture of a prime which splits completely; i.e., $f=1, g=2, e=1$. A path $R$ of length 4 is shown in the tetrahedron. There are 2 prime cycles $R^{\prime}, R^{\prime \prime}$ in the cube above $R$, each with the same length as $R$.

The graph theory Chebotarev density theorem (see Theorem 3 below) says that the density of primes of $X$ with $f=1$ is $\frac{1}{2}$ while that with $f=2$ is also $\frac{1}{2}$.

Definition 10. Let $C$ be a path in $X$ that starts at vertex a of $X$. Given $\lambda \in \operatorname{Gal}(Y / X)$ the path $C$ lifts to a unique path $\widetilde{C}$ in $Y$ of the same length as $C$ starting at $(a, \lambda)$; suppose the lift ends at $(a, \sigma)$. If $D$ represents a prime of $Y$ over $C$ that starts at $(a, \lambda)$ and has the residual degree $f$, then $D$ is the unique lifting of $C^{f}$ to $Y$. The Frobenius automorphism of the prime $[D]$ 

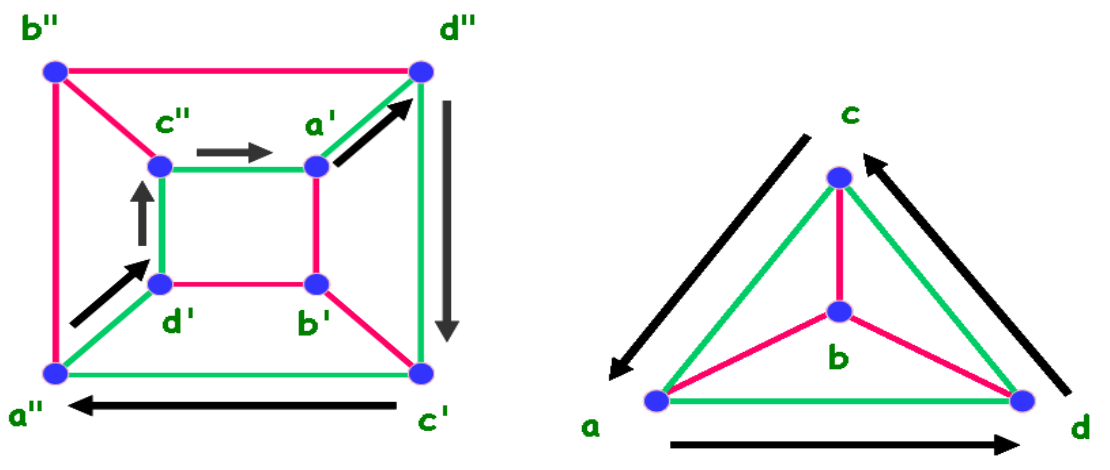

FIGURE 5. Picture of splitting of prime with $f=2, g=1, e=1$. There is 1 prime cycle $P^{\prime}$ in the cube above $P$ in $K_{4}$ and $P^{\prime}$ is the lift of $P^{2}$.

is the Galois group element taking sheet $\lambda$ to sheet $\sigma$; i.e.,

$$
[Y / X, D]=\sigma \lambda^{-1} \in \operatorname{Gal}(Y / X) .
$$

For any path $C$, in $X$, starting at vertex a, the normalized Frobenius automorphism is $\sigma(C)=\sigma$, where $C$ lifts to a unique path $\widetilde{C}$ in $Y$ of the same length as $C$ starting at $(a, 1)$ and ending at $(a, \sigma)$. Here 1 denotes the identity in $\operatorname{Gal}(Y / X)$.

\section{Example 9. Frobenius Automorphisms for the Primes in the Last Example.}

Consider again the cube over the tetrahedron. Computing the Frobenius of either of the primes in the cube in Figure 4 yields the trivial element of the Galois group, while the Frobenius of the prime in Figure 4 is the non-trivial element of the Galois group.

The proof of the following proposition is not hard. See Proposition 2 in Part II of [14].

\section{Proposition 2. Some Properties of the Frobenius Automorphism.}

Suppose $Y / X$ is a normal graph covering. Then we have the following facts.

1) The Frobenius is independent of the choice of $D$ in the equivalence class $[D]$.

2) If $\tau \in \operatorname{Gal}(Y / X)$, then $[Y / X, \tau \circ D]=\tau[Y / X, D] \tau^{-1}$.

Suppose $Y / X$ is normal with abelian Galois group $G$. Then any irreducible representation $\rho$ of $G$ is a character (i.e., a one-dimensional representation); i.e., if $\mathbb{T}$ is the multiplicative group of complex numbers of absolute value 1, $\rho: G \rightarrow \mathbb{T}$ is a group homomorphism. Let $\widehat{G}$ be the set of all these characters. Then $|\widehat{G}|=|G|$. See [15] for more information on representations of finite groups.

Definition 11. Assume that $Y / X$ is a normal graph covering with abelian Galois group G. The Artin L-function for $\rho \in \widehat{G}$ and $u \in \mathbb{C}$ with $|u|$ sufficiently small is

$$
L(u, \rho, Y / X)=\prod_{[C] \text { prime }}\left(1-\rho([Y / X, D]) u^{v(C)}\right)^{-1},
$$

where we recall (3.1) defining the Frobenius. The product is over primes $[C]$ of $X$, with $[D]$ any prime of $Y$ over $[C]$.

The following two definitions give an analog of Definition 6 .

Definition 12. $R_{\rho}$ is the diagonal $2 m \times 2 m$ matrix with diagonal entry for oriented edge e given by $\rho(\sigma(e))$.

Definition 13. $W_{\rho}=u R_{\rho} W_{0}$, where $W_{0}$ is the 0,1 edge matrix from Definition 4.

Theorem 2. Properties of the Artin L-Function for Abelian Coverings.

Assume $Y / X$ is normal with abelian Galois group $G$.

1) If $\rho=1$, the trivial character, then the L-function is the ordinary Ihara zeta function:

$$
L(u, 1, Y / X)=\zeta(u, X) .
$$

2) If $Y / X$ is normal with abelian Galois group $G$, the Ihara zeta function of $Y$ factors as a product of Artin L-functions:

$$
\zeta(u, Y)=\prod_{\rho \in \widehat{G}} L(u, \rho) .
$$


Here the product is over all $\rho \in \widehat{G}$.

3) $L(u, \rho, Y / X)^{-1}=\operatorname{det}\left(I-W_{\rho}\right)$.

Before stating the Chebotarev density theorem for abelian covers, we need a definition. Here we are imitating the discussion Stark gives of the Chebotarev theorem in number fields in [13].

Definition 14. If $R$ denotes the closest pole (necessarily real) of $\zeta(u, X)$ to the origin and $S$ is a set of primes in $X$, define the analytic density $\delta(S)$ to be

$$
\begin{aligned}
\delta(S) & =\lim _{u \rightarrow R^{-}}\left(\frac{\sum_{[C] \in S} u^{\nu(C)}}{\sum_{[C]} u^{v(C)}}\right)=\lim _{u \rightarrow R^{-}}\left(\frac{\sum_{[C] \in S} u^{\nu(C)}}{\log \zeta(u, X)}\right) \\
& =\lim _{u \rightarrow R^{-}}\left(\frac{\sum_{[C] \in S} u^{v(C)}}{-\log (R-u)}\right) .
\end{aligned}
$$

Here the sums are over primes $[C]$ in $X$ and the limit is taken over real values of $u$ less than $R$.

Theorem 3. Graph Theory Chebotarev Density Theorem for Abelian Coverings.

Suppose the graph $X$ is not a cycle graph. If $Y / X$ is abelian and $g$ is a fixed element in the Galois group $G=G(Y / X)$

$$
\delta\{[C] \text { prime of } X \mid \sigma(C)=g\}=\frac{1}{|G|} .
$$

Here $\sigma(C)$ is the normalized Frobenius for $C$ from Definition 10.

The proofs of these things (for general, not necessarily abelian, covers) can be found in [17] and Part II of [14]. You can find analogs of all the properties of the usual Artin $L$-functions listed in Lang [10]. For example, one has the usual induction property for the induced representation of $\operatorname{Gal}(Y / X)$ from a representation of a subgroup. It is this property which is essential for the proof of Theorem 2 .

We define some matrices associated to character $\rho$ of $G$, where $Y / X$ is a normal covering with abelian Galois group $G$.

Definition 15. For $\sigma, \tau \in G$ and vertices $a, b$ of $X$, define the $A(\sigma, \tau)$ matrix to be the $|X| \times|X|$ matrix given by setting the entry $A(\sigma, \tau)_{a, b}=$ the number of directed edges in $Y$ from $(a, \sigma)$ to $(b, \tau)$. Here every undirected edge of $Y$ has been given both directions.

One sees easily that

$$
A(\sigma, \tau)=A\left(1, \sigma^{-1} \tau\right) .
$$

Thanks to formula (3.2), we may define the $|X| \times|X|$ matrix $A(\tau)$ by

$$
A(\tau)=A(1, \tau) .
$$

Definition 16. If $Y / X$ is a normal covering with abelian Galois group $G, \rho \in \widehat{G}$ and $A(\sigma)$ is given by Definition 15 along with formulas (3.2) and (3.3), define the $\rho$ modified adjacency matrix $A_{\rho}$ to be the $|X| \times|X|$ matrix:

$$
A_{\rho}=\sum_{\sigma \in G} \rho(\sigma) A(\sigma) .
$$

Theorem 4. Diagonalization of the Adjacency Matrix of an Abelian Normal Cover. Suppose that $Y / X$ is normal with abelian Galois group $G$. Let $\widehat{G}$ be the complete set of characters of $G$. Then, the adjacency matrix of $Y$ is similar to a block diagonal matrix with diagonal entries $A_{\rho}$, as $\rho$ runs through $\widehat{G}$.

Proof. Here we use facts from Terras [15]. The adjacency operator on $Y$ may be viewed as a coming from the induced representation $I n d_{\{e\}}^{G} 1$. To see this, list the vertices of $Y$ as $(x, \tau), x \in X, \tau \in G$. This decomposes $A_{Y}$ into $|X| \times|X|$ blocks, with blocks given by Definition 15. Now formula (3.2) says that $A(\sigma, \tau)=A\left(\sigma^{-1} \tau\right)$, for $\sigma, \tau \in G$. This means $\sigma \in G$ is acting on the function $A: G \rightarrow \mathbb{R}$ via $\lambda(\sigma) A(\tau)=A\left(\sigma^{-1} \tau\right)$, for $\sigma, \tau \in G$. By definition, $\lambda$ is the left regular representation of $G$. This is equivalent to the representation $\operatorname{Ind}_{\{e\}}^{G} 1$ on $G$ induced up from the trivial representation on the subgroup $\{e\}$, where $e$ is the identity of $G$. 
A basic fact from representation theory (see p. 256 of [15]) says that when $G$ is abelian we have the decomposition:

$$
\operatorname{Ind}_{\{e\}}^{G} 1 \cong \sum_{\rho \in \widehat{G}} \oplus \rho .
$$

Here the symbol $\cong$ means that the 2 representations are equivalent; i.e., one is obtained from the other by uniform change of basis. The direct sum on the right is just the diagonal matrix formed from the $\rho \in \widehat{G}$. It follows from formula (3.4) that $A_{Y}$ is similar to a diagonal matrix with entries $A_{\rho}$ corresponding to $\rho \in \widehat{G}$.

Now we have an $L$-function analog of formula (2.3).

Theorem 5. Ihara Theorem for Vertex Artin L-Function.

Assume $Y / X$ normal with abelian Galois group $G$. For $\rho \in \widehat{G}$, we have

$$
L(u, \rho, Y / X)^{-1}=\left(1-u^{2}\right)^{r-1} \operatorname{det}\left(I-A_{\rho} u+Q u^{2}\right) .
$$

Here $A_{\rho}$ is the $\rho$ modified adjacency matrix, $Q$ is the diagonal degree -1 matrix, $r$ is the rank of the fundamental group of $X$.

Proof. The Bass proof works just as in the weighted graph case of the previous section. See Part II of [14] or [17].

Example 10. The Cube over the Tetrahedron. For our example in Figure 3, the Galois group $G(Y / X)=\{1, \sigma\}$. We write $a^{\prime}=(a, 1)$ and $a^{\prime \prime}=(a, \sigma)$, for $a \in X$. The representations of the Galois group are $\widehat{G}=\{1, \rho\}$, where $\rho(\sigma)=-1$.

Then the matrices are

$$
A(1)=\left(\begin{array}{llll}
0 & 1 & 0 & 0 \\
1 & 0 & 1 & 1 \\
0 & 1 & 0 & 0 \\
0 & 1 & 0 & 0
\end{array}\right) \text { and } A(\sigma)=\left(\begin{array}{cccc}
0 & 0 & 1 & 1 \\
0 & 0 & 0 & 0 \\
1 & 0 & 0 & 1 \\
1 & 0 & 1 & 0
\end{array}\right)
$$

So

$$
A_{1}=\left(\begin{array}{llll}
0 & 1 & 1 & 1 \\
1 & 0 & 1 & 1 \\
1 & 1 & 0 & 1 \\
1 & 1 & 1 & 0
\end{array}\right)=\text { the adjacency matrix of } X
$$

and

It follows that

$$
A_{\rho}=\left(\begin{array}{rrrr}
0 & 1 & -1 & -1 \\
1 & 0 & 1 & 1 \\
-1 & 1 & 0 & -1 \\
-1 & 1 & -1 & 0
\end{array}\right)
$$

$$
\begin{aligned}
\zeta_{Y}(u) & =L(u, \rho) \zeta_{X}(u), \\
L(u, \rho)^{-1} & =\left(1-u^{2}\right)(1+u)(1+2 u)\left(1-u+2 u^{2}\right)^{3} \\
\zeta_{X}(u)^{-1} & =\left(1-u^{2}\right)^{2}(1-u)(1-2 u)\left(1+u+2 u^{2}\right)^{3} .
\end{aligned}
$$

You can view these formulas as providing a factorization of an $8 \times 8$ determinant as a product of two $4 \times 4$ determinants.

In a subsequent paper we will use graph coverings of directed graphs to explain the factorization of $\zeta_{K_{4}}(u)^{-1}$, which does not cover anything in the usual sense of the word "cover." See Horton [7] for more information on Ihara zeta functions of directed graphs. Irregular examples involving much larger determinants can also be worked out. See the end of Section 3 of Stark and Terras [14].

Next consider some larger examples and the distributions of roots of the Ihara zeta functions involved.

Example 11. $\mathbb{Z}_{m} \times \mathbb{Z}_{n}$-Cover of 2-Loops Plus Vertex.

Note that if $g . c . d .(m, n)=1$, then $\mathbb{Z}_{m} \times \mathbb{Z}_{n}$ is cyclic by the Chinese remainder theorem. Our first example is a $\left(\mathbb{Z}_{m} \times \mathbb{Z}_{n}\right)$ cover of the graph which consists of 2 loops with an extra vertex on 1 loop. Figure 6 shows the case $m=n=3$.

We compute the edge L-functions associated to the characters $\chi_{r, s}(x, y)=\exp \left(2 \pi i\left[\frac{r x}{m}+\frac{s y}{n}\right]\right)$. Look at the base graph in Figure 6. We lift edge $a$ up a unit in the $x$-coordinate; i.e., the normalized Frobenius $\sigma(a)=(1,0)$, and we lift edge $b$ up a unit in the $y$-coordinate; i.e., $\sigma(b)=(0,1)$. Figure 7 shows the poles of the Ihara zeta function for the $\left(\mathbb{Z}_{63} \times \mathbb{Z}_{64}\right)$ - cover of the base graph in Figure 6. The Riemann hypothesis is visibly very false.

For our next example, we consider a random cover of the same base graph.

Example 12. Random Cover of 2 Loops with an Extra Vertex on 1 Loop. 

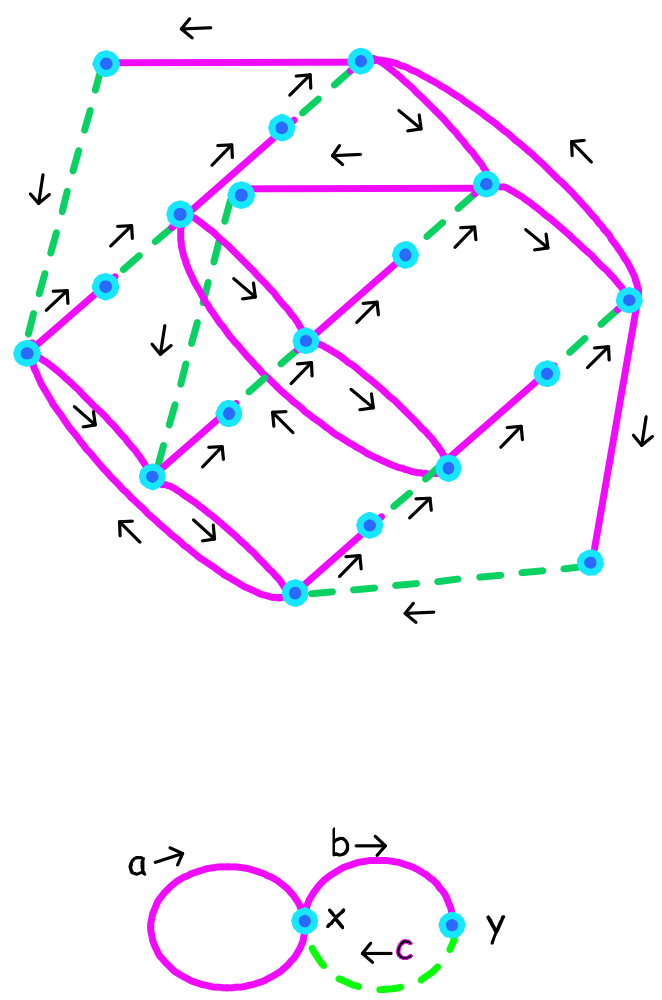

FiguRE 6. The top graph is a $\mathbb{Z}_{3}^{2}$ - cover of 2 loops with an extra vertex on one loop. The spanning tree below is a dashed line. The sheets above are drawn in the same way. It appears to be a torus graph with some extra decoration.

To produce figures analogous to Figure 7 for a random cover of the base graph consisting of 2 loops with an extra vertex on one of them, we can use the formula for the edge matrix $W_{0}$ of the covering graph in terms of the start matrix $S$ and the terminal matrix $T$ from Proposition 1 above. It is also convenient to write $S=(M N), T=(N M)$ where $M$ and $N$ have $|E(Y)|$ columns. It follows that

$$
W_{0}=\left(\begin{array}{cc}
{ }^{t} N M & { }^{t} N N-I \\
{ }^{t} M M-I & { }^{t} M N
\end{array}\right) .
$$

Now arrange the columns of $M$ so that the columns corresponding to lifts of a given edge of the base graph are listed in order of the sheet on which the lift starts. And the lifts of a given vertex of the base graph are also listed together in the order of the sheets where they live. Then if $d$ is the number of sheets of our cover,

$$
M=\left(\begin{array}{ccc}
I_{d} & I_{d} & 0 \\
0 & 0 & I_{d}
\end{array}\right) \text { and } N=\left(\begin{array}{ccc}
A & 0 & I_{d} \\
0 & B & 0
\end{array}\right),
$$

where $A$ and $B$ are permutation matrices. Suppose the number of sheets of the cover is $d=3$ and the lift of edge $a$ corresponds to the permutation (12) while the lift of edge $b$ corresponds to the permutation (13) in the symmetric group $S_{3}$ of permutations of the 3 sheets. Then we get the graph in Figure 8.

We used Matlab to plot the reciprocals of the eigenvalues of $W_{0}$ for covers in which $A$ and $B$ are random permutation matrices (found using the command randperm in Matlab). When $d=700$, we obtain Figure 9. If we compare this with the picture found by Angel, Friedman, and Hoory [1] for random covers of the base graph $K_{4}^{\prime}$, which is $K_{4}-e d g e$, remembering that they are plotting the reciprocals of the poles of the Ihara zeta function of the covering graph, we see that there is much similarity, though their $R$ is $2 / 3$, while ours is approximately .4694 .

When we compare poles in the last 2 examples, we find some similarities. Both regions look 2 dimensional. Neither looks uniformly distributed on the unit circle. The region inside the green circle of radius $\sqrt{R}$ is what we call the "non-Riemann Hypothesis region" - except for $R$ (and possibly $-R$ ). There is not much there for the random cover. The worst exceptional (i.e., non-Riemann hypothesis and not from the base graph) poles in Figure 9 are the four mysterious real poles near the circle of radius $\sqrt{R}$. They appear in Figure 9 but sometimes, in similar experiments, we see only the left two exceptional real poles, sometimes only the two on the right, and sometimes no exceptional real poles. 


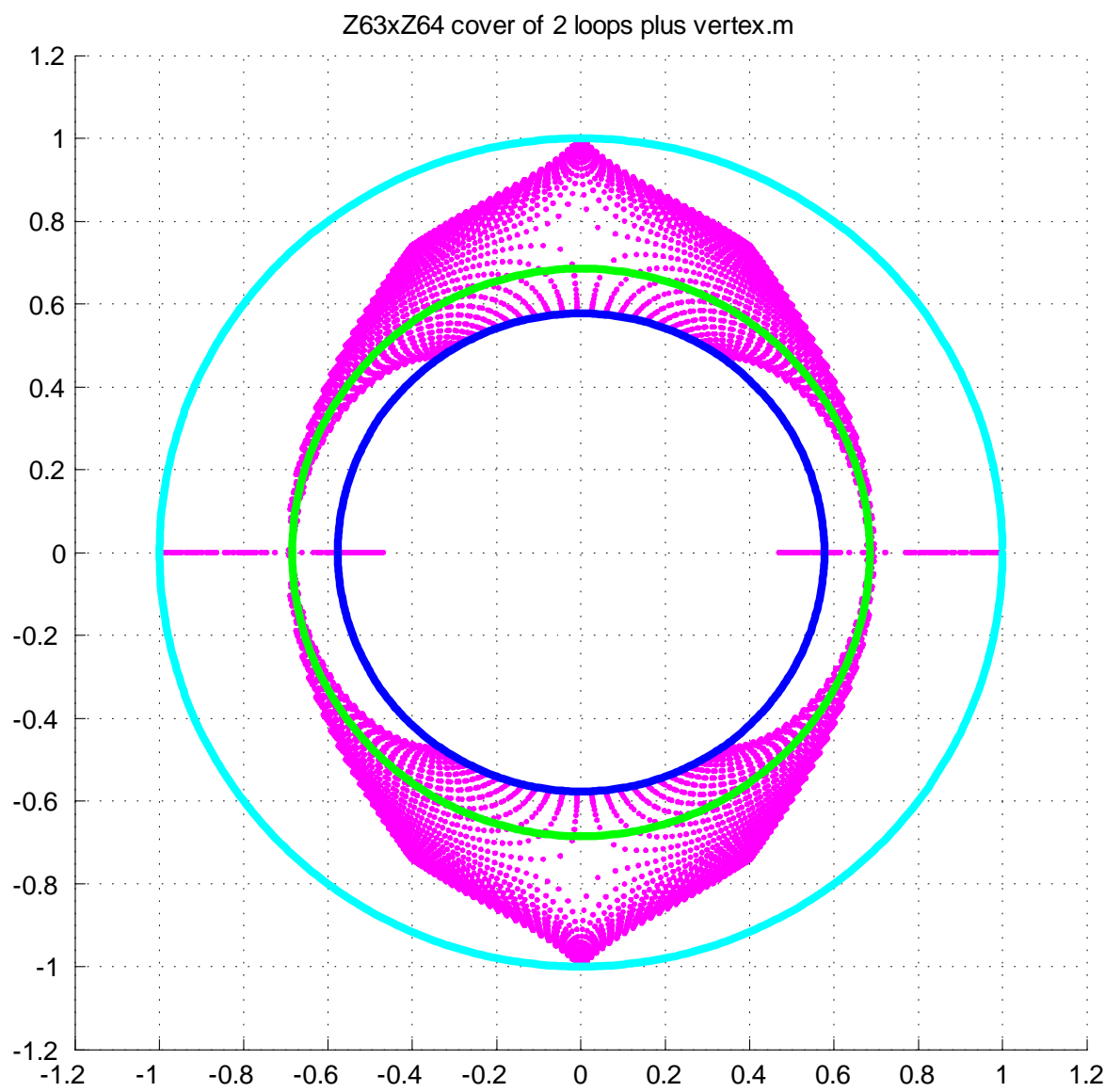

FIGURE 7. The points are the poles of the Ihara zeta function for a $\mathbb{Z}_{63} \times \mathbb{Z}_{64}$ - cover of 2 loops plus a vertex on 1 loop. The circles are centered at the origin and have radii $\frac{1}{\sqrt{q}}, \sqrt{R}, \frac{1}{\sqrt{p}}$, where $q=3, p=1$, and the closest pole to the origin $R$ is approximately 0.4694 . The Riemann hypothesis is very false as there are many poles well inside the circle of radius $\sqrt{R}$.

So one could conjecture that the Riemann hypothesis is approximately true for a large random cover. However the abelian cover has lots of bad poles. By a theorem of Kotani and Sunada [9], the non-real poles of the Ihara zeta function must lie between circles of radius $p^{-1 / 2}$ and $q^{-1 / 2}$, where $p+1=$ minimum degree and $q+1=$ maximum degree.

Question: Let $X$ be your favorite irregular base graph. Is there any family of groups $G_{n}$, with $\left|G_{n}\right| \rightarrow \infty$, as $n \rightarrow \infty$, with Galois coverings $Y_{n} / X$ having Galois group $G_{n}$ such that the zeta functions $\zeta\left(Y_{n}, u\right)$ satisfy the Riemann hypothesis; i.e., all poles $u \neq R$ satisfy $|u| \geq \sqrt{R}$ ? The graphs $Y_{n}$ could perhaps be viewed as "Ramanujan covers" in the spirit of Lubotzky, Phillips, and Sarnak [11]. 

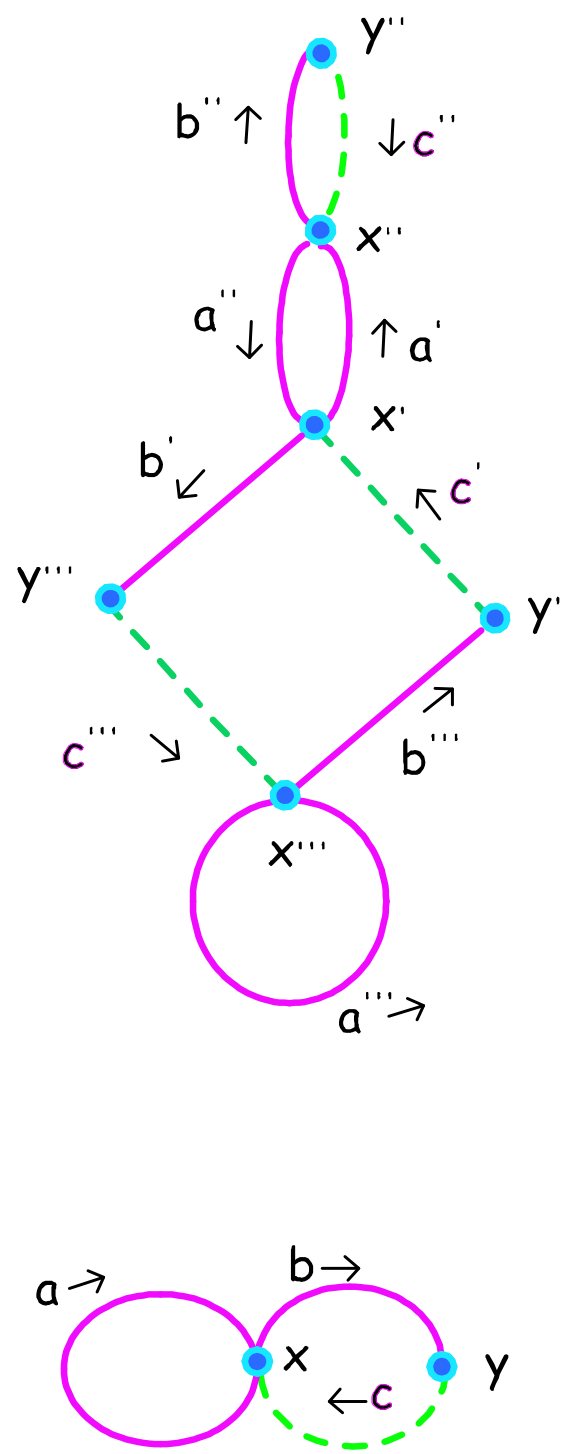

FIGURE 8. The random 3-cover of 2 loops with and extra vertex with the lift of $a$ corresponding to the permutation (12) and the lift of $b$ corresponding to the permutation (13). The dashed line in the base graph is the spanning tree and the dashed lines in the cover are the sheets of the cover (from top to bottom in the order: sheet 2 , sheet 1 , sheet 3 ). 


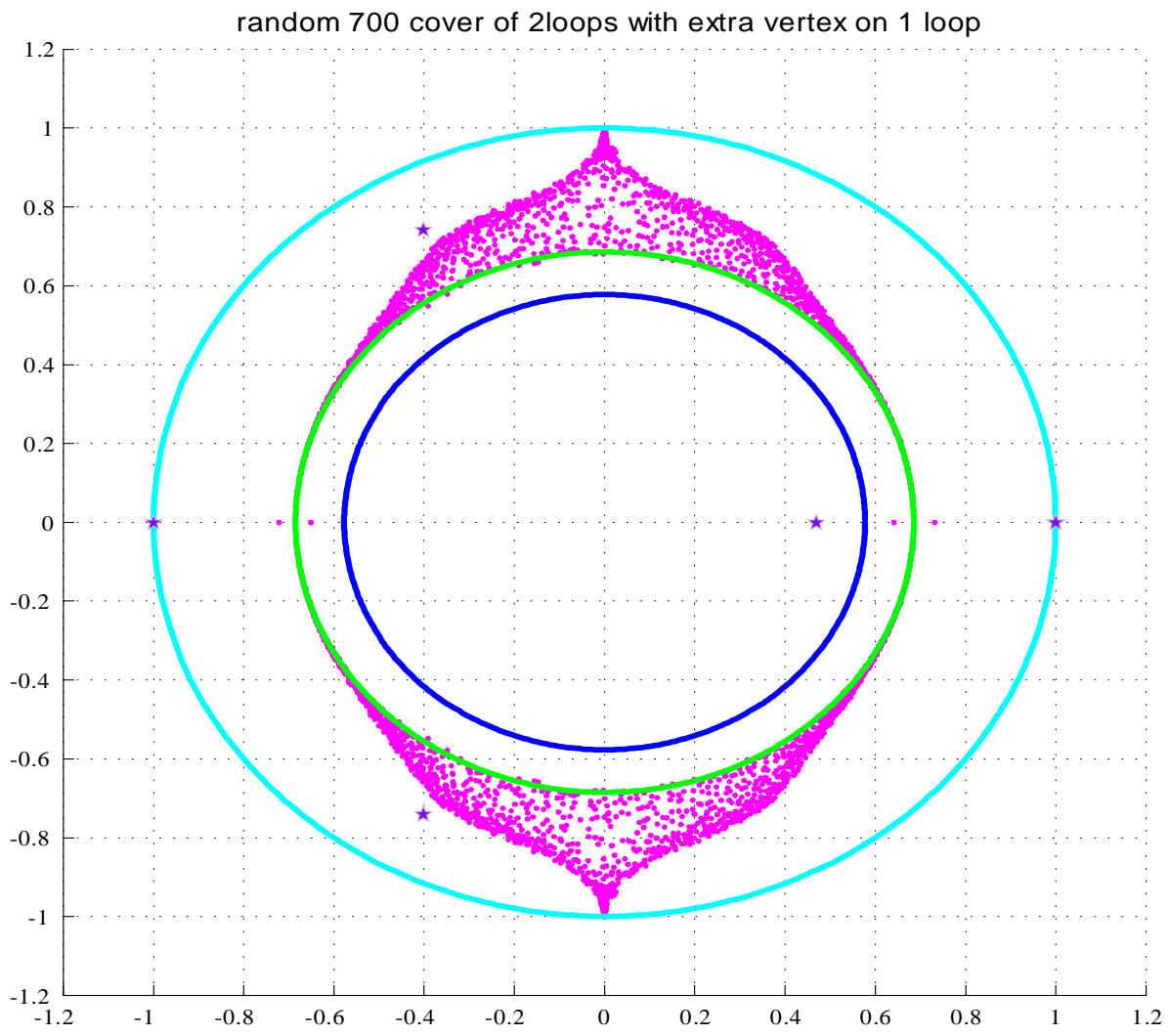

FIGURE 9. The points are poles of the Ihara zeta function of a random 700 cover of the graph consisting of 2 loops with an extra vertex on one of the loops. The stars are the poles which are also poles of the zeta function of the base graph. The circles are centered at the origin and have radii $\frac{1}{\sqrt{q}}, \sqrt{R}, \frac{1}{\sqrt{p}}$, where $q=3, p=1$, and the closest pole to the origin $R$ is approximately 0.4694 . The Riemann hypothesis looks approximately true as very few poles $u \neq R$ are inside the circle of radius $\sqrt{R}$ and the poles inside this circle are very close to the circle except for 4 real poles. The new (i.e., not from the base graph) real non-Riemann hypothesis poles seem to appear sporadically. Sometimes they are only on the left, sometimes only on the right, sometimes missing altogether. 


\section{REFERENCES}

[1] O. Angel, J. Friedman, and S. Hoory, The non-backtracking spectrum of the universal cover of a graph, preprint.

[2] Z. D. Bai, Circular Law, Ann. Prob., 25, (1997), 494-529.

[3] H. Bass, The Ihara-Selberg zeta function of a tree lattice, Internatl. J. Math., 3 (1992), 717-797.

[4] J. Friedman, A proof of Alon's second eigenvalue conjecture, Memoirs of the A.M.S., to appear.

[5] G. Berkolaiko, R.Carlson, S.Fulling, and P.Kuchment, Proceedings of the Joint Summer Research Conference on Quantum Graphs and Their Applications, Contemporary Math., 415, Amer. Math. Soc., Providence, 2006.

[6] K. Hashimoto, Zeta functions of finite graphs and representations of p-adic groups, Adv. Studies in Pure Math., Vol. 15, Academic Press, N.Y., 1989, pp. 211-280.

[7] M. Horton, Ihara zeta functions of digraphs, preprint.

[8] M. D. Horton, H. M. Stark, A. A. Terras, What are zeta functions of graphs and what are they good for?, in Contemporary Math., 415, Amer. Math. Soc., Providence, 2006, pp. 173 to 190.

[9] M. Kotani and T. Sunada, Zeta functions of finite graphs, J. Math. Sci. Univ. Tokyo, 7 (2000), 7-25.

[10] S. Lang, Algebraic number theory, Addison-Wesley, Reading, MA, 1968.

[11] A. Lubotzky, R. Phillips, and P. Sarnak, Ramanujan graphs, Combinatorica, 8 (1988), 261-277.

[12] U. Smilansky, Quantum chaos on discrete graphs, preprint.

[13] H. M. Stark, Galois theory, algebraic number theory and zeta functions, in From Number Theory to Physics, M. Waldschmidt et al. (Eds.), Springer-Verlag, Berlin, 1992.

[14] H. M. Stark and A. A. Terras, Zeta Functions of Finite Graphs and Coverings, I, II, III, Advances in Math., 121 (1996), 124-165; 154 (2000), 132-195; 208 (2007) 467-489.

[15] A. A. Terras, Fourier Analysis on Finite Groups and Applications, Cambridge Univ. Press, Cambridge, U.K., 1999.

[16] A. A. Terras, Arithmetical quantum chaos, in IAS/Park City Math. Series, Vol. 12 (2007), 333-375.

[17] A. A. Terras, a stroll through the garden of graph zeta functions, at the website: www.math.ucsd.edu/ aterras/newbook.pdf.

Math. Dept., Wellesley College

MATh. DePT., U.C.S.D.

MATh. DePT., U.C.S.D.

E-mail address: aterras@ucsd.edu

URL: http://www.math.ucsd.edu/ aterras 